\title{
Wakefield damping in a pair of $X$-band accelerators for linear colliders
}

\author{
Roger M. Jones, ${ }^{*}$ Christopher E. Adolphsen, Juwen W. Wang, and Zenghai Li \\ Stanford Linear Accelerator Center, 2575 Sand Hill Road, Menlo Park, California 94025, USA
}

(Received 28 July 2006; published 2 October 2006)

\begin{abstract}
We consider the means to damp the wakefield left behind ultrarelativistic charges. In particular, we focus on a pair of traveling wave accelerators operating at an $X$-band frequency of $11.424 \mathrm{GHz}$. In order to maximize the efficiency of acceleration, in the context of a linear collider, multiple bunches of charged particles are accelerated within a given pulse of the electromagnetic field. The wakefield left behind successive bunches, if left unchecked, can seriously disturb the progress of trailing bunches and can lead to an appreciable dilution in the emittance of the beam. We report on a method to minimize the influence of the wakefield on trailing bunches. This method entails detuning the characteristic mode frequencies which make up the electromagnetic field, damping the wakefield, and interleaving the frequencies of adjacent accelerating structures. Theoretical predictions of the wakefield and modes, based on a circuit model, are compared with experimental measurements of the wakefield conducted within the ASSET facility at SLAC. Very good agreement is obtained between theory and experiment and this allows us to have some confidence in designing the damping of wakefields in a future linear collider consisting of several thousand of these accelerating structures.
\end{abstract}

DOI: 10.1103/PhysRevSTAB.9.102001

PACS numbers: $41.75 . \mathrm{Ht}$, 41.75.Fr

\section{INTRODUCTION}

In the design of a future linear collider we are required to accelerate bunches of charged particles to a center of mass of the order of $1 \mathrm{TeV}$ [1]. One means of achieving this end is with $X$-band technology in which the accelerating structures operate at room temperature and accelerate bunches of charged particles at a frequency of $11.424 \mathrm{GHz}$. Furthermore, in order to efficiently accelerate the particles, it is required to accelerate many bunches within a given pulse of the rf accelerating field. In the NLC (next linear collider) design, we envisaged accelerating 192 bunches; each bunch consisting of $10^{10}$ electron or positrons. Until comparatively recently [2], this means of providing acceleration of particle in a linear collider was on equal footing with another method, namely, TESLA [3] which accelerates particles within superconducting niobium cavities. The latter technology choice (although there is considerable design optimization available within this decision) has been adopted by a consensus of the international community as the one to conduct a concerted effort on the design of a complete linear collider [1]. However, over a decade and a half of experience and the lessons learned in the design of the NLC will be invaluable in aiding the design of the superconducting linear collider. Moreover, the design principles that are employed for the NLC main linac structures are essentially valid for any accelerator which requires a low emittance and high current beam. Furthermore, the damping scheme may have some relevance to the Compact

\footnotetext{
*Author to whom correspondence may be addressed: Department of Physics and Astronomy, The University of Manchester, Oxford Road, Manchester, M13 9PL, United Kingdom.

Electronic address: roger.jones@manchester.ac.uk
}

Linear Collider (CLIC [4]), which is still proceeding with a full design at an acceleration frequency of $30 \mathrm{GHz}$, and similar to the NLC, the wakefield is forced to partially decohere by detuning individual cells of each accelerating structure.

Here we describe the design of damping the wakefield that trails behind each bunch and we report on recent experiment made at the ASSET (Accelerator Structure SETup) facility of the SLAC linear collider and compare it with theoretical predictions. Prior to proceeding with a description of the approach used in designing the damping of the wakefield, it is important to point out how we decided upon the particular structure to accelerate the particle beams in the first place. Initially we chose a traveling wave accelerating structure with a phase advance of $2 \pi / 3$ per cell as this has the largest shunt impedance (a measure of the efficiency of conversion of $\mathrm{rf}$ power into beam power [4]) and it has been shown to be successful in accelerating the beam on the 2 mile SLAC linac. However, over the past few years we have discovered that highgradients $(65 \mathrm{MV} / \mathrm{m}$ prior to acceleration of beam) have resulted in significant breakdown within the interior body of the structure. In order to ameliorate this condition, we opted for a design in which the electromagnetic (e.m.) field of the fundamental accelerating mode undergoes a phase advance of $5 \pi / 6$ per cell. This results in a lower group velocity of the accelerating mode. Furthermore, we adopted a ratio of the average iris to free-space wavelength of $a / \lambda \sim 0.17$. Both of these design choices were motivated by experimental evidence that the breakdown in the structure is kept to acceptable levels under these conditions [5]. We are still left with the decision as to how many cells will be fed by the input power coupler. In our design we chose 55 cells. This choice was motivated by two condi- 
tions, namely, cost and electrical breakdown considerations. In order to minimize the overall cost of each structure, we are required to maximize the number of cells each coupler feeds. However, we are also constrained by the power that can be fed into each coupler, without there being breakdown within the vicinity of the coupler and in the interior of the cells. The power fed into each structure increases with structure length as the power is dissipated in the form of heat on the $\mathrm{Cu}$ walls and in losing energy to the accelerated beam along the structure. For this reason we limit the length of each structure to be approximately $60 \mathrm{~cm}$ and this constrains the number of cells to be 55 for a structure operating in the $5 \pi / 6$ accelerating mode.

We now return to the main topic of this paper, namely, the wakefield left behind the accelerated particles within the beam. The wakefield can be decomposed into a series of multipoles [6]. The first multipole (other than the $m=0$ accelerating mode of course) is the most dangerous mode as far as disturbing the progress of the beam down the linac is concerned. This multipole is known as the dipole component of the wakefield and herein we limit ourselves to damping this particular multipole only. The other higher order multipoles decay more rapidly with a radial dependence of $r^{m}$ and for small transverse offsets they can be safely neglected as they contribute little to overall transverse force the beam experiences. We distinguish between two regimes in the wakefield: the intrabunch wakefield and the long-range wakefield. The former constitutes the wakefield seen by particles seen within a particular bunch and the latter corresponds to the wakefield left behind a bunch and is experienced by trailing bunches. The intrabunch, or short-range wakefield can shown to be proportional to the fourth power of the average iris radius $\left(a^{4}\right.$ [7]) and this, in addition to the reason dictated by minimization of electrical breakdown outlined earlier, is the reason we chose $a / \lambda \sim 0.17$ as this gives manageable wakefields. On the other hand, the long-range wakefield is damped by several means. The initial damping of the wake is affected by forcing the modes which constitute the wakefield to not add coherently. This is achieved by adiabatically changing the dimensions of the iris radius down the structure such that it gets smaller as the beam proceeds downstream towards the high energy end of the structure. The beam is maintained in the $5 \pi / 6$ mode of acceleration by retuning the diameter of each gap adjacent to each iris in the appropriate way. This is all done with a chosen set of fiducial cells from the structure, in precise detail using the finite element code OMEGA3P [8] and the parameters of intermediate cells are obtained by spline interpolation. The specific functional dependence of iris radius on cell number is discussed in the following section. Also, in addition to tailoring the dimensions of the irises of each structure we force the wakefield to fall even more rapidly initially by meshing the frequencies of neighboring structures such that their dipole frequencies are interleaved.

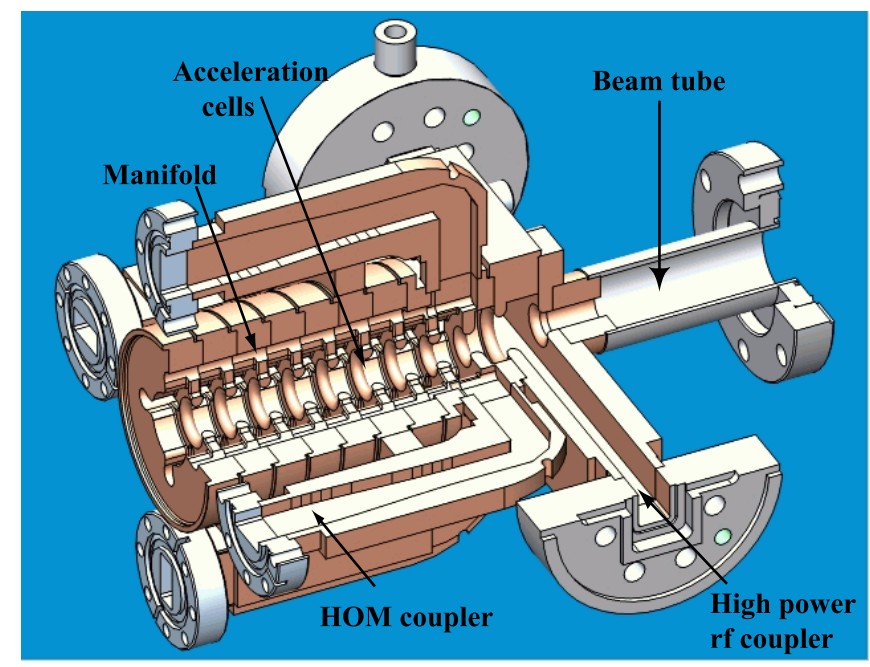

FIG. 1. (Color) CAD drawing illustrating a cross-sectional view of the output region of H60VG4SL17A through slots present in almost all of the 55 cells of the structure. The $Q$ associated with this damping is approximately 500 and we refer to this as a medium damped structure (in contradistinction to, for example, the CLIC structure, which is heavily damped with a $Q \sim 10$ $[9,10])$.

However, damping the wake by this means is not adequate as eventually the wakefield recoheres and consequently starts to rise to unacceptable levels, from the point of view of beam dynamics. This is mitigated by providing damping four manifolds which run along the outer walls of each accelerating structure. The manifolds and accelerating structure are illustrated in Fig. 1. These manifolds intercept a portion of the wakefield by coupling it out.

This paper is organized such that the following section describes the theory behind how the wakefield is calculated in the structure. The third section describes the issues that arise in fabricating these structures very precisely and a comparison is made with the theoretical predictions on the frequency of the modes. The fourth section discusses the experimental measurement of the wakefield on a pair of accelerating structures and this is compared with theoretical predictions. The consequences of this wakefield on the dynamics of the beam are discussed in the penultimate section. Some conclusions of this research are discussed in the final section.

\section{WAKEFIELD DAMPING: THEORY AND DESIGN}

In order to calculate the overall wakefield of a multicell accelerating structure, we utilize a circuit model with input parameters taken from a limited set of cells and their dispersion properties are simulated with very precise finite element [8] and finite difference [11] computer codes. The reason we do not use a finite element code such as OMEGA3P [8] to calculate the wakefield of the complete 55-cell structure is that it would require a substantial 
amount of computing time and disk space to model it properly. Furthermore, at the design stage of the accelerator project it is crucially important to be able to routinely make rapid adjustments to the geometrical parameters and feed these back to the wakefield model in order to ascertain the impact on the beam dynamics. Once the parameters of the circuit have been fully parametrized, it is an extremely rapid process to calculate the wakefield utilizing the circuit model as the parameters are subsequently varied. Also, at the time of the design of the accelerator, the complete wakefield for the entire structure from a finite element code was not available [12].

\section{A. Circuit model of dipole modes coupled to waveguide manifold}

The circuit model for three out of the complete set of 55 cells is illustrated in Fig. 2. In the circuit, inductive coupling is utilized between the TM-TM modes and TE-TE modes and the cross-coupled TM-TE modes (and vice versa). This particular form of coupling was chosen based upon investigations with MAFIA [11] and OMEGA3P simulations of the e.m. field in the region of the iris of a single cell. However, there is no loss in generality, as changing the sign of the associated coupling coefficient changes the

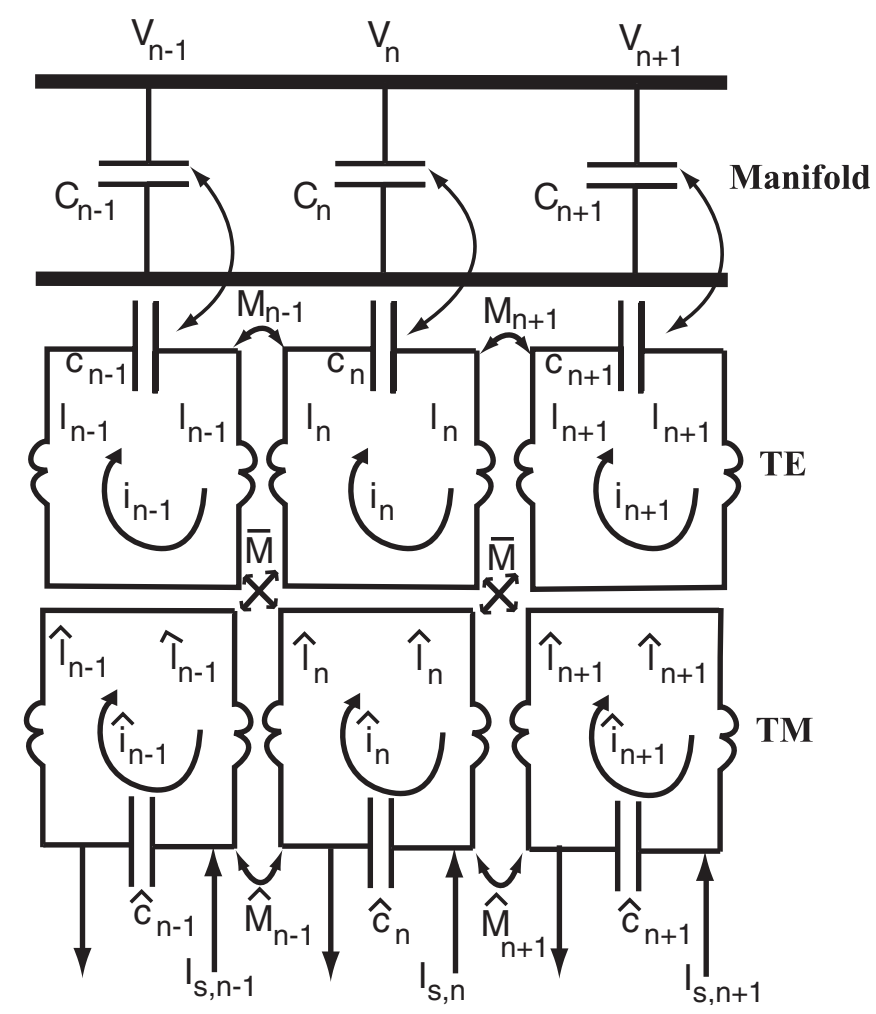

FIG. 2. Circuit diagram illustrating the hybrid TE-TM dipole mode (represented by the parallel $L-C$ circuits) coupled to the waveguide modes (represented uppermost by the transmission line) for three cells of the main accelerating structure. The currents $I_{s, n-1}, I_{s, n}$, and $I_{s, n+1}$ represent the delta-source electron beam which is coupled directly into the lowermost TM circuit. effective coupling to capacitive coupling. The mutual coupling between adjacent TM modes in the circuits is $M$, the corresponding one for the TE circuits is $\hat{M}$ and the crosscoupling term is $\bar{M}$. The manifold is designed such that a $\mathrm{TE}_{10}$ mode propagates within it. Thus, we allow for capacitive coupling between the cell modes and the manifold. The circuit equation corresponding to this coupling at an angular frequency $\omega(=2 \pi f)$ is given by

$$
\begin{aligned}
& V_{n}=-j\left(I_{n} / C_{n}+i_{n} \kappa_{n} / \sqrt{C_{n} c_{n}}\right) / \omega \\
& v_{n}=-j\left(i_{n} / c_{n}+I_{n} \kappa_{n} / \sqrt{C_{n} c_{n}}\right) / \omega .
\end{aligned}
$$

Here $\left(C_{n}, c_{n}\right)$ represents the manifold shunt capacitance and TE cell capacitance, respectively, $\left(V_{n}, \boldsymbol{v}_{n}\right)$ the corresponding voltages across them, and $\left(I_{n}, i_{n}\right)$ the currents through them. Thus the dimensionless quantity $\kappa_{n}$ provides the manifold-to-cell coupling. It is to be noted that the hybrid nature of the dipole mode is such that the TM/TE ratio becomes larger as one proceeds downstream the cells of a particular structure and thus the coupling coefficient $\kappa_{n}$ needs to increase correspondingly in order to obtain an approximately fixed damping value. The network equations for the circuit in Fig. 2 can be written in the form given below:

$$
\begin{aligned}
R A & =G a \\
\left(H-1 / f^{2}\right) a+H_{x} \hat{a}=G A & =G R^{-1} G a \\
\left(\hat{H}-1 / f^{2}\right) \hat{a}+H_{x}^{t} a & =B / f^{2} .
\end{aligned}
$$

Here the $n$th components of the above column vectors $a_{n} \quad\left(=i_{n} / \sqrt{c_{n}}\right), \quad \hat{a}_{n} \quad\left(=\hat{i}_{n} / \sqrt{\hat{c}_{n}}\right), \quad$ and $A_{n} \quad\left(=\frac{j V_{n}}{f} \times\right.$ $\sqrt{\frac{c}{P}}\left[z \operatorname{sinc} \phi_{0 n}\right]^{-1 / 2}$, where $\phi_{0 n}$ is phase advance per waveguide section and $z$ is the characteristic impedance of the waveguide section) are proportional to the loop currents $\left(i_{n}\right)$ in the TE circuits, the loop currents $\left(\hat{i}_{n}\right)$ in the TM circuits, and the shunt voltages $\left(V_{n}\right)$, in the manifolds, respectively. There are $N(=55)$ components to each of these vectors. $B$ is also an $N$-component vector proportional to the driving currents. All matrices $H, \hat{H}, H_{x}$ are tridiagonal as only the coupling of neighboring cells is taken into account in the circuit analysis. The matrix $R$ describes the manifold and is also tridiagonal and $G$, a diagonal matrix, is a direct function of the coupling of the TE manifold mode to the TE component of the dipole mode - both of which are $N \times N$ matrices. The cell matrixes $H, \hat{H}, H_{x}$ describe the TE, TM, and cross-coupled TE-TM mode, respectively. The latter has vanishing diagonal elements as it describes cross-coupling terms only. The nonzero elements of all these matrices have the following form:

$$
\begin{gathered}
R_{n n}=-2 \cos \phi_{n}, \quad R_{n n \pm 1}=1 \\
\cos \phi_{n}=\cos \phi_{0 n}-\alpha_{n}(\pi P f / c)^{2} \operatorname{sinc} \phi_{0 n}
\end{gathered}
$$




$$
\begin{gathered}
\phi_{0 n}=(2 \pi P / c) \sqrt{f^{2}-F_{c n}^{2}} \\
H_{n n}=1 / f_{n}^{2} \\
H_{n n \pm 1}=\eta_{n \pm 1 / 2} /\left(2 f_{n} f_{n \pm 1}\right) \\
H_{x, n n \pm 1}= \pm \eta_{x, n \pm 1 / 2} /\left(2 f_{n} \hat{f}_{n \pm 1}\right) \\
\hat{H}_{n n}=1 / \hat{f}_{n}^{2}, \quad \hat{H}_{n n \pm 1}=-\hat{\eta}_{n \pm 1 / 2} /\left(2 \hat{f}_{n} \hat{f}_{n n \pm 1}\right) \\
G_{n n}=\Gamma_{n}(\pi P / c) \sqrt{\operatorname{sinc} \phi_{0 n}} .
\end{gathered}
$$

In Eq. (9) we have used the definition: $\operatorname{sinc} x=\sin x / x$. There are eight $n$-dependent parameters per cell exhibited in the above equations. We discuss them below. The quantity $\phi_{n}$ represents the local phase advance per section of the manifold regarded as a quasiperiodic structure with slowly varying properties. Two parameters are required for its description: $F_{c}$ corresponding to the cutoff of the local waveguide section, and $\alpha=\left(2 C\left[P C_{T}\right]^{-1}\right.$, where $C_{T}$ is the capacitance per unit length of transmission line) to the ratio of the shunt capacitance to the capacitance of the transmission line. The $n$-independent parameter $P$ is the section length, describing the periodicity of the system and for our structure is equal to five-twelfths of the wavelength of the accelerating field. In writing Eqs. (3) and (4) we have omitted terms second order in the section-tosection variation. The quantities $f_{n}\left(=\left[2 \pi \sqrt{l_{n} c_{n}}\right]^{-1}\right), \hat{f}_{n}$ ( $\left.=\left[2 \pi \sqrt{\hat{l}_{n} \hat{c}_{n}}\right]^{-1}\right)$ correspond to the resonant frequencies of the TE and TM component of the cells. The three coupling coefficients, TE-TE, TM-TM, and crosscoupled TM-TE are given in terms of the mutual inductances indicated in Fig. 2 as: $\eta_{n \pm 1 / 2}=M_{n \pm 1}\left[\sqrt{l_{n} l_{n \pm 1}}\right]^{-1}$, $\hat{\eta}_{n \pm 1 / 2}=\hat{M}_{n \pm 1}\left[\sqrt{\hat{l}_{n} \hat{l}_{n \pm 1}}\right]^{-1}, \quad$ and $\quad \eta_{n \pm 1 / 2}=$ $\bar{M}_{n \pm 1}\left[\sqrt{l_{n} \hat{l}_{n \pm 1}}\right]^{-1}$, respectively. The eighth parameter, $\Gamma_{n}$ $\left(=\sqrt{\alpha_{n} \kappa_{n}^{2}}\left[1-\kappa_{n}^{2}\right]^{-1}\right)$, represents the $n$th manifold-to-TE coupling coefficient. It is a function of $\alpha$ and $\kappa$ and it is used for convenience in place of the previously defined coupling coefficient $\kappa_{n}$.

With $\Gamma_{n}$ set equal to zero, the cell equations become identical to those of detuned accelerator structure described in [13], and the explicit connection of the relevant parameters to the circuit can be found there. This is to be expected, as in this case the circuit represents nothing more than an accelerator in which the dipole modes are detuned and there is no manifold loading at all (effectively an infinite $Q$ ). The source vector $B$ in Eq. (2c) is expressed in terms of cell kick factors in precisely the same manner as in Secs. 2.3 and 3.2 of [13]. Also we follow [13] in terminating the chain of cells as implied by simply requiring, $n, n \pm 1$ to all belong to the set $\{1, \ldots, N\}$. In this paper, we terminate the manifold with an outgoing wave boundary condition, which can be shown to require that we replace $R_{11}$ and $R_{N N}$ by $-\exp \left(j \phi_{1}\right)$ and $-\exp \left(j \phi_{N}\right)$, respectively. For most purposes we eliminate the explicit appearance of the manifold amplitude vector $A$ by using Eq. (2a) to bring the right-hand side (RHS) of Eq. (2b) to the second form shown. In order to obtain the 8 parameters of each cell we specialize to a structure consisting of identical and infinitely repeating cells and we construct Brillouin diagrams for each of these cells. The parameters of a particular cell are then extracted from fits to the curves. We do this for a limited set of cells - 12 out of a possible 55 . The additional parameters are obtained by spline interpolation. This procedure is outlined in the following section.

\section{B. Brillouin diagram of a structure consisting of infinitely periodic cells}

The parameters for a particular cell are determined by fitting to the frequency versus phase advance dispersion curves of the three lowest modes of strictly periodic structures having the dimensions of that particular cell. These are determined from OMEGA3P frequency domain simulations of a single section with specified phase advance boundary conditions. The dispersion relation is obtained from Eqs. (2) with the definitions in Eqs. (3)-(9), utilizing the periodicity condition that all mode vectors vary in the from $b_{n}=b \exp (j n \psi)$ and is readily obtained as

$$
\begin{aligned}
& \left\{\left[(1+\eta \cos \psi) / f_{0}^{2}-f^{-2}\right]\left[(1-\hat{\eta} \cos \psi) / \hat{f}_{0}^{2}-f^{-2}\right]-\eta_{x}^{2} /\left(f_{0} \hat{f}_{0}\right) \sin ^{2} \psi\right\}[\cos \psi-\cos \phi] \\
& \quad=\Gamma^{2}(\pi P / c)^{2}\left[(1-\hat{\eta} \cos \psi) \hat{f}_{0}^{-2}-f^{-2}\right] \operatorname{sinc} \phi
\end{aligned}
$$

In Eq. (10) $f_{0}\left(\hat{f}_{0}\right)$ represents the TE (TM) cell resonant frequency. Also, $\eta(\hat{\eta})$ is the corresponding TE (TM) coupling coefficient and $\eta_{x}$ the cross-coupling coefficient. As an example of the procedure, we illustrate its application to cell 27 in Fig. 3(a). The parameters are determined by requiring that the three curves pass through eight selected phase-frequency pairs (the red dots in Fig. 3) determined from simulations. The 0 and $\pi$ phase advance points provide four pairs, and four points near the avoided crossing provide the rest. The dashed curves are obtained by setting the RHS of Eq. (10) to 0. They represent the dispersion curves of the lower and upper dipole modes and of the manifold mode when direct manifold-cell coupling is suppressed. The black dots in Fig. 3(a), also from simulations, are used to assess the quality of the fit rather than for parameter determination. Effective damping is 

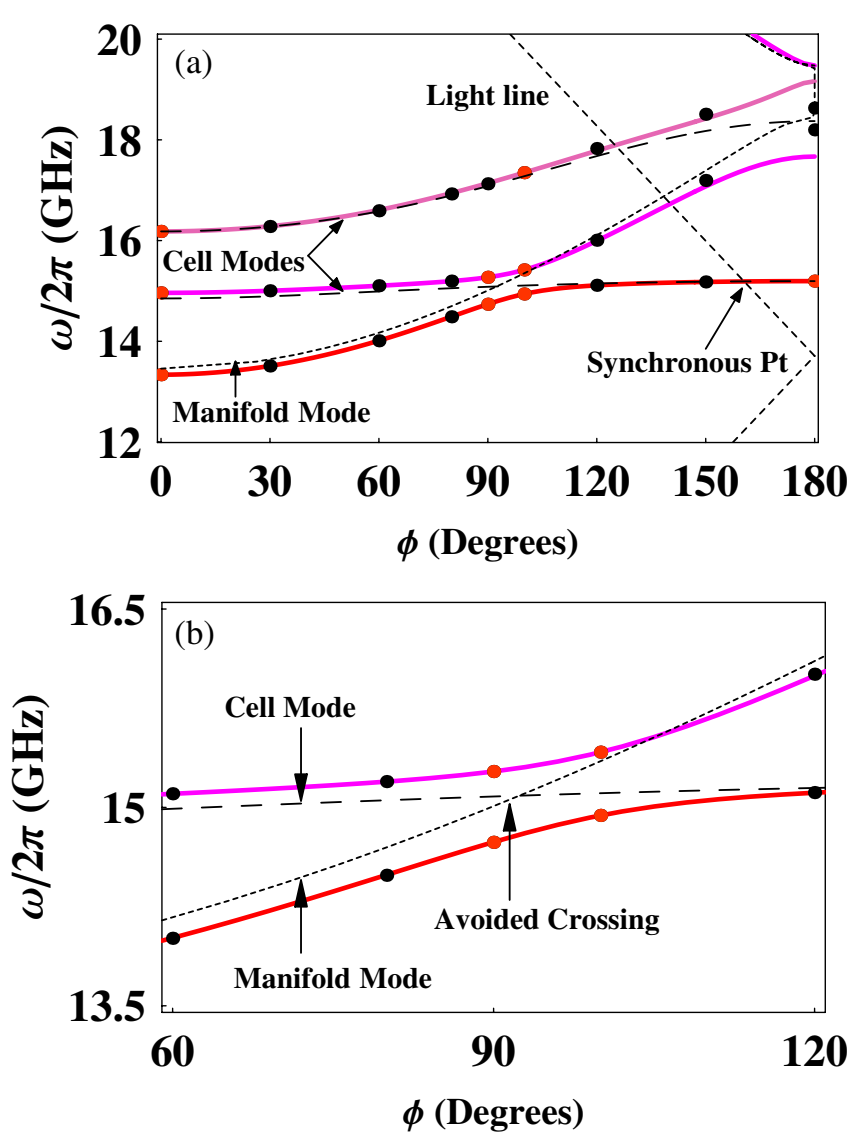

FIG. 3. (Color) (a) Brillouin diagram for cell 27 of a 55-cell structure. The dashed curves represent the dispersion curves for the modes prior to coupling to the manifold. Also illustrated is the light line and the point at which it intersects the cell mode (in which the beam is synchronous with the dipole mode). (b) Expanded view of the characteristic manner in which the manifold mode and the cell mode avoid crossing due to the coupling of the modes. The strength of the coupling is proportional to the minimum degree of separation of the coupled modes.

associated with the crossing of the lower dipole mode and the manifold mode. When the coupling is included the crossing is avoided as shown by the solid curves (and shown in an expanded view in Fig. 3(b) for clarity). The coupling gets stronger as the minimum separation of the avoided crossing of the modes gets larger and we change the cell geometrical parameters accordingly, to achieve the desired avoided crossing and associated damping $Q$ value.

\section{Formalism of determination of wakefield from the spectral function method}

Prior to determining the wakefield we recast Eq. (2) in matrix form:

$$
\left(\begin{array}{cc}
\hat{H} & H_{x}^{t} \\
H_{x} & H-G R^{-1} G
\end{array}\right)\left(\begin{array}{l}
\hat{a} \\
a
\end{array}\right)-f^{-2}\left(\begin{array}{l}
\hat{a} \\
a
\end{array}\right)=f^{-2}\left(\begin{array}{c}
B \\
0
\end{array}\right) .
$$

The diagonal elements of $H, G$, and $R$ are frequency dependent. To further condense the notation we may also write Eq. (11) in $2 N$ by $2 N$ matrix form:

$$
\bar{H} \bar{a}-f^{-2} \bar{a}=f^{-2} \bar{B} .
$$

The drive beam, represented by the $N$ component vector $B$, couples only to the TM mode. We take it to be a point charge moving at velocity $c$ and normalize it per unit charge per unit displacement. The final wakefield will be that corresponding to excitation by a delta-function source. To obtain the wakefield of a realistic beam distributionusually assumed to be Gaussian - the point source wakefield may be convolved with the spatial distribution [14]. With this understanding, the Fourier transform of the deltafunction source term takes the form

$$
B_{n}=\sqrt{\left(4 \pi f_{s}^{n} / c\right) K_{s}^{n} P} \exp [-(j 2 \pi f / c) n],
$$

where $K_{s}^{n}$ is the single-cell kick factor [13] evaluated at the synchronous mode and $f_{s}^{n}$ the synchronous mode frequency, both evaluated for a strictly periodic structure based upon the $n$th cell [13]. The transverse wake function (i.e. wake potential per unit length) for a particle trailing a distance $s$ behind a velocity $c$ drive bunch (per unit drive bunch charge per unit drive bunch displacement) may be written

$$
W_{t}(s)=\int[Z(f-j \varepsilon) \exp [(2 \pi j s / c)(f-j \varepsilon)] d f,
$$

where $\varepsilon$ is a positive infinitesimal quantity and the wake impedance $Z$ is given by

$$
Z(f)=\pi^{-1} \sum_{n, m}^{N} \sqrt{K_{s}^{n} K_{s}^{m} f_{s}^{n} f_{s}^{m}} \exp [(2 \pi j L / c) f(n-m)] \tilde{H}_{n m}
$$

with the $2 N$ by $2 N$ matrix $\tilde{H}$ given by

$$
\tilde{H}=\bar{H}\left(1-f^{-2} \bar{H}\right)^{-1} .
$$

From causality $Z(f)$ can be analytically extended to the lower half plane (LHP), and singularities on the real axis are avoided in Eq. (14) by integration over $f$ just below the real axis as indicated in Eq. (14). Because $W$ is real, we also have $Z(f)=Z^{*}\left(-f^{*}\right)$, for $f$ in the LHP. Because $Z$ is real for sufficiently low frequencies on the real axis, $Z^{*}\left(f^{*}\right)$ provides an analytic extension of $Z$ into the upper half plane. Since the $Z$ so defined is discontinuous across the real axis where $Z$ is complex, cuts are introduced there to render $Z$ single valued on what we call the "physical sheet" of its Riemann surface. It also satisfies $Z(f)=$ $Z(-f)$, that is, it is an even function of $f$ in the complex plane. We note that $Z$ is actually a four valued function arising from the sign ambiguity in $\sin \phi_{1}$ and $\sin \phi_{N}$, quantities which appear in $R_{11}$ and $R_{N N}$, respectively. [The $\cos \phi_{n}$, defined by Eq. (4) are single valued analytic functions, but the corresponding sines are defined only by the 
trigonometric identity, $\sin ^{2}+\cos ^{2}=1$.] Damped modes appear as complex poles on sheets of the Riemann surface adjacent to the physical sheet.

Because the equivalent circuit wake function contains a small nonphysical precursor on the $[-N L, 0]$ interval [13], it proves to be convenient to define a "causal" wake function by

$$
W_{c}(s)=\theta(s)\left[W_{t}(s)-W_{t}(-s)\right] .
$$

$W_{c}$ equals $W t$ for $s>N L$ and vanishes for negative $s$. In the interval $[0, N L] W_{t}(-s)$ would be zero in the absence of a precursor. Hence Eq. (17) represents a smooth way of suppressing the precursor, and $W_{c}$ is more likely to portray the actual structure than the strict equivalent circuit model. From Eq. (14) and the symmetry properties of $Z$ noted in the previous section, we have

$$
W_{t}(-s)=\int_{-\infty}^{\infty} Z(f+j \varepsilon) \exp [(2 \pi j s / c)(f+j \varepsilon)] d f,
$$

which leads to

$$
\begin{array}{r}
W_{t}(s)-W_{t}(-s)=2 j \int_{-\infty}^{\infty} \operatorname{Im}\{Z(f-j \varepsilon)\} \\
\times \exp [(2 \pi j s / c) f] d f \\
=4 \int_{0}^{\infty} \operatorname{Im}\{Z(f+j \varepsilon)\} \sin [(2 \pi j s / c) f] d f .
\end{array}
$$

To include the contribution of poles on the real axis (with real residue) in Eqs. (19) and (20) we interpret

$$
\operatorname{Im}\left\{\left(f \pm j \varepsilon-f_{0}\right)^{-1}\right\}=\mp \pi \delta\left(f-f_{0}\right)
$$

and define $4 \operatorname{Im}\{Z(f+j \varepsilon)\}$ as the spectral function $S(f)$ of the wake function. Thus, we have

$$
W_{c}(s)=\theta(s) \int_{0}^{\infty} S(f) \sin [(2 \pi s / c) f] d f .
$$

It is usually more convenient to display the maximum excursions in the amplitude of the field, otherwise known as the envelope of the wakefield. It is straightforward to obtain the envelope of the wakefield from Eq. (22) as

$$
W_{c}(s)=\theta(s)\left|\int_{0}^{\infty} S(f) \exp [(2 \pi s j / c) f] d f\right| .
$$

For the undamped case, corresponding to a purely detuned structure [13], we set the coupling matrix $G$ to zero, $Z$ is real on the real axis and contains a set of poles on the real axis at the modal frequencies. The spectral function is then simply a sum of delta functions:

$$
S(f)=2 \sum_{p} K_{p} \delta\left(f-f_{p}\right)=2 K_{n} d n / d f
$$

where the $f_{p}$ are the modal frequencies, $n(f)$ is the number of modes with frequency less than $f$, and the $K_{p}$ are called modal kick factors. The spectral function and the modal sum methods are thus formally identical. In the presence of damping, $Z$ is complex on those portions of the real axis which lie in the propagation bands of the manifolds, and poles which would lie on that portion of the real axis in the absence of coupling to the manifold split into complex conjugate pairs on the nonphysical sheets accessed by analytic continuation through the cuts. When the coupling is weak so that their position can be found by perturbation theory, their distance from the real axis is small compared to their separation, and the spectral function has sharp narrow peaks in place of the delta functions of the undamped case. As the coupling strength increases, these poles move further from the real axis, the peaks broaden, and while the peaks generally remain quite discernible, the behavior is relatively smooth. The spectral function can be computed as a function of frequency by direct evaluation of Eq. (15). In the weak coupling case it is relatively simple to determine the modal frequencies, eigenvectors and $Q$ values and hence to compute the damped modal sum. In contrast, a large number of frequency points is required to adequately delineate the narrow peaked spectral function. The situation is reversed in the strong coupling case. The process of determining the eigenmodes is a difficult and computationally time consuming process as there are $3 \times N$ modes to be determined which must be carefully distinguished from each other, while on the other hand the number of frequency points required to adequately describe the more smoothly varying spectral function becomes more reasonable. Finally, we note that the wakefield given in Eq. (22), the causal wakefield, will be used in all subsequent calculations.

The application of the spectral function technique to the calculation of the wakefield for a set of interleaved spectral functions is outlined in the following section.

\section{Evaluation and optimization of wakefield in practice}

After the geometrical parameters have been defined, the associated circuit parameters are calculated and the impedance is calculated from Eq. (15). First, we note that in practice the impedance is not calculated from Eq. (11) [or Eq. (12) for that matter]. Since the matrices are all decidedly sparse (as they are at most tridiagonal) it proves to be substantially more computationally efficient to solve the below matrix equation:

$$
\left(\begin{array}{ccc}
\hat{H} f^{2}-U & H_{x}^{t} & 0 \\
H_{x} & H f^{2}-U & 0 \\
0 & G & -R
\end{array}\right)\left(\begin{array}{l}
\hat{a} \\
a \\
A
\end{array}\right)=\left(\begin{array}{l}
B \\
0 \\
0
\end{array}\right),
$$

where $U$ is the identity matrix of dimension $N \times N$. Furthermore, wall losses are introduced into the analysis by providing a resistor in series with each of the $L-C$ circuits and also in the transmission line which represents 
the manifold. This adds an imaginary term to the diagonals of the corresponding $\mathrm{H}$-matrices, but it has the benefit of broadening the associated resonant peaks in the spectral function and thus reduces the required sampling of frequency points.

The optimal spectral distribution is determined by the rms of the sum wakefield, the standard deviation of the sum wakefield, and by the wake at the first trailing bunch. The sum wake is defined as the wakefield at a particular bunch summed at the location of all previous bunches. In this way, the rms wakefield indicates whether or not beam breakup (BBU) is likely to occur and the standard deviation of the sum wake allows tolerances on alignments of cell-to-cells and groups thereof to be calculated. In all cases it is important to minimize these quantities. To this end, a Fortran computer code OPTIMIZESPECTRALWAKE was written which minimizes the sum of these three quantities as the bandwidth of the distribution and associated standard deviation is varied. We found that the wakefield decayed in an optimal manner when an initial distribution of the form $K d n / d f \propto[\operatorname{sech} f]^{1 / 2}$ is used.

In addition, we interleaved the frequencies of 2 structures such that the peaks in one spectral function were located as best they could be between the peak of the spectral function of the neighboring structure. However, in order to facilitate the rapid fabrication of the pair of structures, we neglected to use this optimal design in the pair of structures in the final design. We chose to locate the synchronous (or cell) frequencies at the intermediate point between those in the neighboring structure. This is illustrated in Fig. 4. This is by no means optimal, as will be seen in the calculation of the overall wakefield from its two spectral components. These spectral components are illustrated in Fig. 5, together with their arithmetic mean, the overall spectral function. The peaks corresponding to the

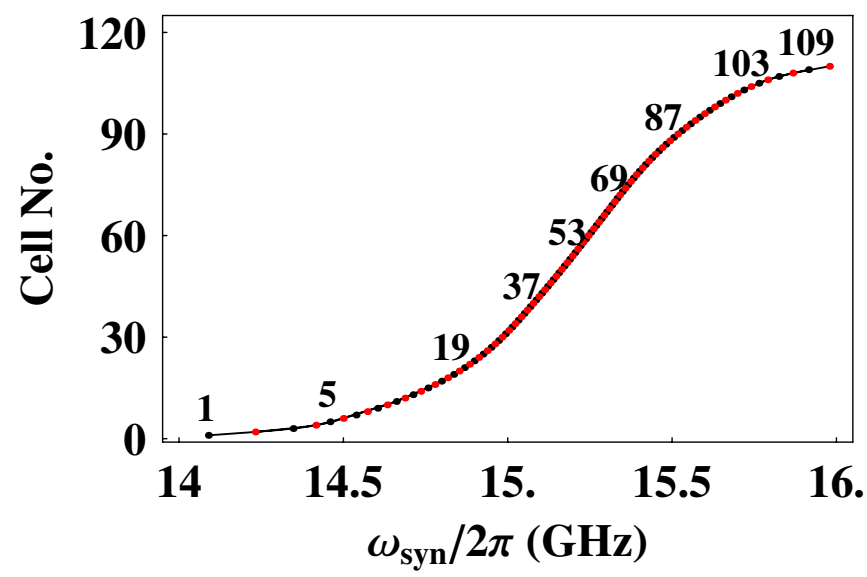

FIG. 4. (Color) Cell number versus synchronous frequency for the first dipole band of a pair of 55 cell structures known as H60VG4SL17A/B. The black points indicate the first structure and the red points the second structure.

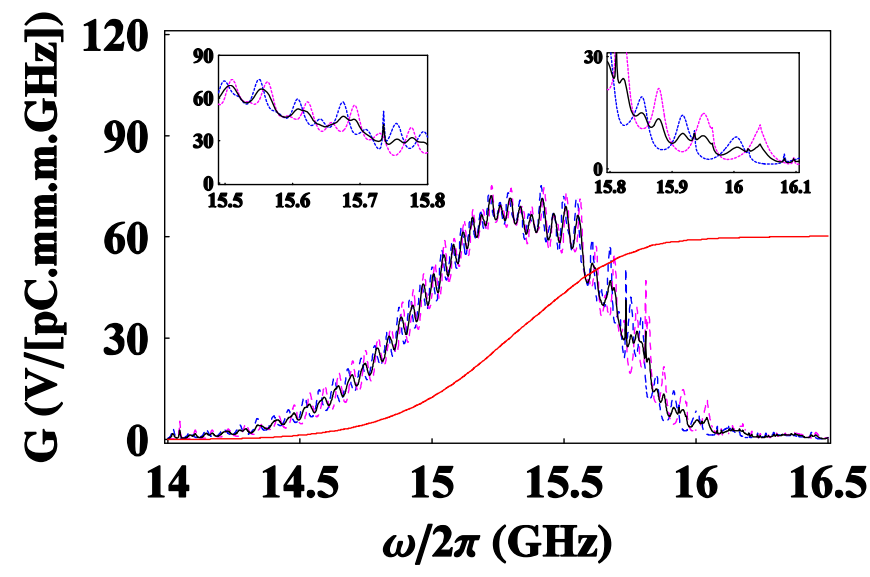

FIG. 5. (Color) Spectral functions of two interleaved accelerator structures: H60VG4SL17A/B shown by the magenta and blue dashed curves. Also shown is the summation of both spectral functions by the solid black curve.

resonant modes of the cavities are shown in the inset of Fig. 5. In the region of $16 \mathrm{GHz}$, the peaks are well located as the overall spectral function is quite well smoothed, compared to the individual components. However, the peaks in the approximate region close to $15.5 \mathrm{GHz}$ are not so well positioned and could certainly be improved upon. The wakefield and its individual components are illustrated in Fig. 6. In the central region (at approximately $20 \mathrm{~ns}$ ), the wakefield rises slightly and this is due to the nonoptimized interleaving of adjacent structures in the region of 15.5 to $15.8 \mathrm{GHz}$. Nonetheless, in the interest of making a rapid experimental verification of the theoretical calculations on the wakefield, the parameters for the nonoptimized design were retained. Some of the issues encountered in fabricating two complete 55 cell structures

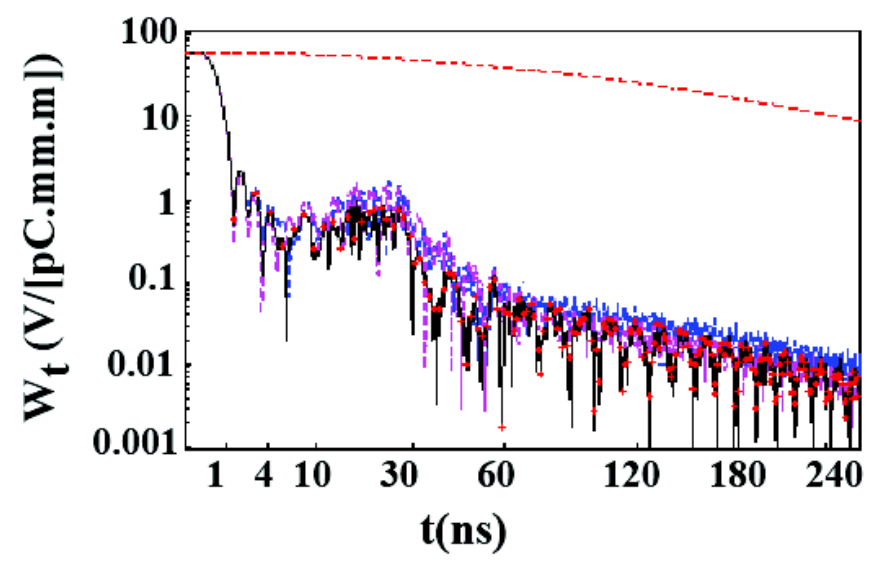

FIG. 6. (Color) . Individual structures are indicated by the magenta and blue dashed curves. Also shown is the summation of both wakefields by the black line. The red dots indicate the $1.4 \mathrm{~ns}$ locations where the bunches are located in an NLC-like design. The red dashed line indicates how the wakefield damps from $\mathrm{Cu}$ losses alone. 
are described in the following section. The various stages employed in the fabrication process and the tolerances achieved in the process of fabricating individual cells are focused upon.

\section{FABRICATION METHODOLOGY AND EXPERIMENTAL MEASUREMENTS ON CELLS}

Based on the design outlined in the previous section, the geometrical features of the cells are faithfully reproduced using a combination of precise milling and a computer numerically controlled lathe equipped with a diamond- tipped cutting element. A typical cell is illustrated in Fig. 7 in which the damping manifolds and coupling slots are shown. Prior to bonding these cells into a complete 55cell stack, the frequencies of individual cells are measured in order to assess how closely the fabricated cells match the initial design frequencies. Both the fundamental, accelerating mode frequency and the dipole-mode frequencies are measured. This was achieved in a custom designed rf measurement device in which we record the frequencies for single-cell phase shifts of 0 and $\pi \mathrm{rad}$. The results of this investigation are shown in Fig. 8. The rms

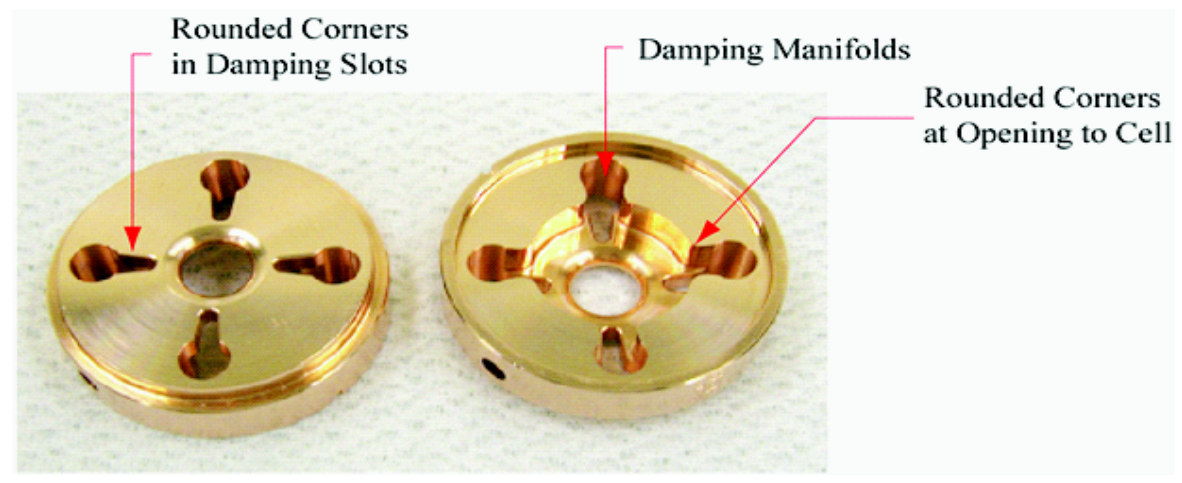

FIG. 7. (Color) Two completed cells for the accelerating structures H60VG4SL17A/B. The inner iris region, where the beam traverses, is surrounded by four manifolds. The tapered coupling slots are clearly displayed in the left-hand photograph.
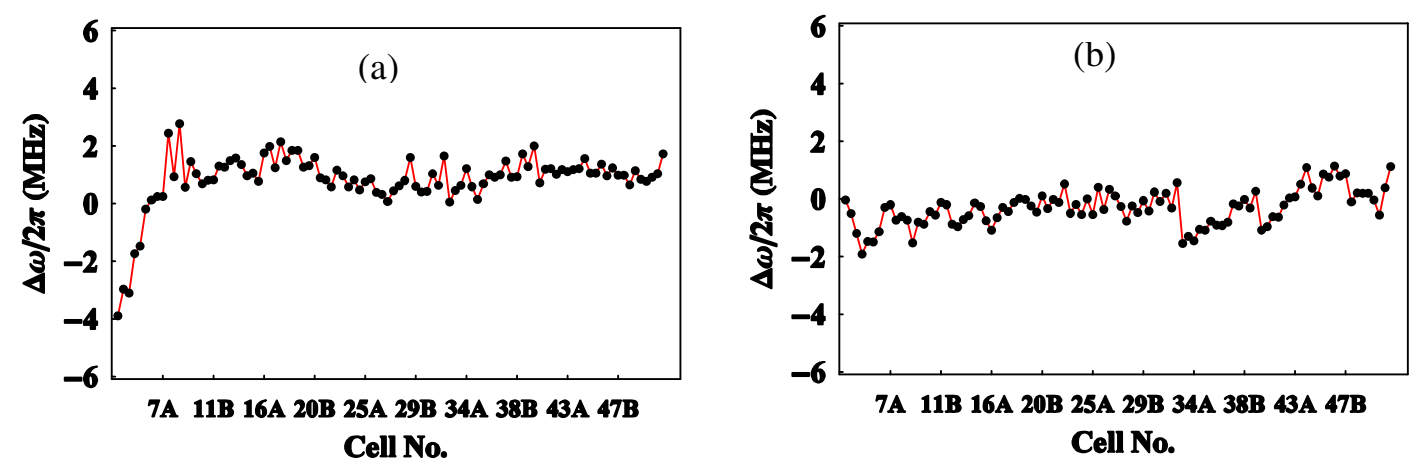

FIG. 8. (Color) Frequency deviations of the fundamental mode (a) and dipole mode (b) frequencies from their design values for all cells of H60VG4SL17A/B.

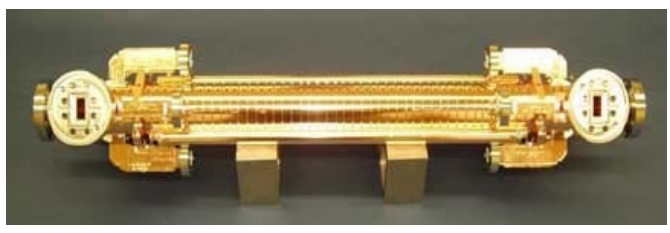

(a)

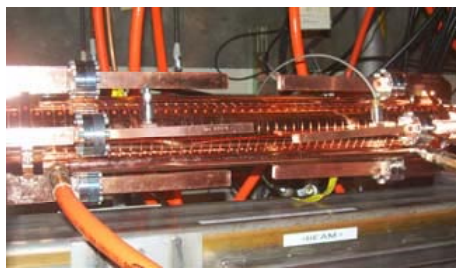

(b)

FIG. 9. (Color) Completed accelerating structure H60VG4SL17A (a) and (b) installed on an alignment strong-back in the ASSET facility of the SLC. 
deviations from smoothly varying curves is approximately $0.6 \mathrm{MHz}$.

This adequately meets the frequency tolerance on the fundamental, which is required to be $1 \mathrm{MHz}$ (which we anticipate to be able to readily correct in the structure by tuning successive cells). Also, the dipole mode is required to smoothly varying to within $3 \mathrm{MHz}$ and thus this wakefield-imposed requirement is also met. Once we were satisfied with the tolerances on the frequencies of the modes, we went ahead and bonded the full structure. This proceeded in two main steps: diffusion bonding of the cells and brazing on the couplers parts. The complete set of cells is carefully aligned on a "v-block" and diffusion bonded in a furnace at a temperature of a little more than $1020^{\circ} \mathrm{C}$. Once we were satisfied with the rigidity of the stack of bonded cells, higher order mode couplers and associated waveguide are subsequently brazed onto the structure in a hydrogen furnace. In order to clean and make the surface ready for high gradient testing, the structures were wet and dry hydrogen fired, then vacuum baked at $650{ }^{\circ} \mathrm{C}$ for 2 to 3 weeks in a double evacuation system. One of a pair of the final accelerator structures, known as H60VG4SL17A, is shown in Fig. 9(a).

After completing the fabrication process a pair (H60VG4SL17A/B) of accelerating structures with interleaved frequencies were subsequently installed in the ASSET facility of the SLAC linac. The beam-induced wakefield measurement on these structures is described in the following section.

\section{EXPERIMENTAL MEASUREMENT OF WAKEFIELD}

The Accelerator Structure SETup (ASSET) wakefield measurement facility is located near the beginning of the SLAC linac, just after the region where damped electrons and positions are injected into it. The facility was constructed in 1994 to measure the $1.8 \mathrm{~m}$ long (206 cell) $X$-band structures that were initially developed for the NLC [15]. Here we describe measurements made with this facility on the pair of $0.6 \mathrm{~m}$ long ( 55 cell) accelerator structures that are the subject of this paper. As described in Sec. II, the dipole-mode frequencies of the pair are interleaved, which improves the deconstructive interference of the beam-induced dipole fields (as if there were one detuned structure of twice the number of cells).
The technique used to measure the transverse wakefield begins with extracting a positron "drive" bunch from the south damping ring and injecting it into the linac via the south ring-to-linac (SRTL) transport line (see Fig. 10). The bunch then traverses the $X$-band structures and is subsequently steered into a dump. The magnet utilized for this purpose serves as the first part of a chicane that transports electrons in the linac back onto the linac axis. The electron bunch itself is extracted from the north damping ring at some later time, $t$, and injected into the linac via the north ring-to-linac transport line. As such, it serves as a "witness" bunch to the wakefield left behind by the positron bunch. From witnessing these fields, the electron bunch is deflected by an angle:

$$
\Delta \phi_{y}=\left[q^{2} n_{d} P e^{-\left[\left(\omega \sigma_{d}\right) /(2 c)\right]^{2}} e^{-\left[\left(\omega \sigma_{w}\right) /(2 c)\right]^{2}} / E_{w}\right] W_{c}(t) \Delta y_{d}
$$

which is proportional to both $W_{c}(t)$, the integrated transverse dipole wakefield at time $t$, and $\Delta y_{d}$, the offset of the drive bunch. The parameters in parentheses consist of: $q$, the charge on an electron, $n_{d}\left(\sim 10^{10}\right)$, the number of particles in the drive bunch, $P(=1.2 \mathrm{~m})$, the active length of the structure pair and $E_{w}(=1.2 \mathrm{GeV})$, the energy of the witness bunch. The exponential factors account for the Gaussian shape of the bunches, which are necessary to include because $W_{c}(t)$ is the field from a pointlike particle rather than a distribution of particles. With the drive and witness bunch lengths $\left(\sigma_{d} \sim \sigma_{w} \sim 0.6 \mathrm{~mm}\right)$ and the central frequency of $\sim 15.2 \mathrm{GH} z$, the product of these exponential terms is approximately 0.9 .

For the vertical wakefield measurements, changes in the vertical deflection of the witness electron bunch were recorded as the positron drive bunch was moved vertically through the structures in a stepwise manner (over about $3 \mathrm{~mm}$ ). The deflection angle of the witness bunch was derived from fits to trajectory data from more than ten beam position monitors, both upstream and downstream of the chicane. The intensity of the drive bunch, i.e., the number of particles, $n_{d}$, was measured pulse-by-pulse as well using a toroid in the SRTL. Once this data was collected, the deflection angle per unit offset and per unit charge of the drive beam was determined. By repeating this procedure at various time separations, the temporal depen-

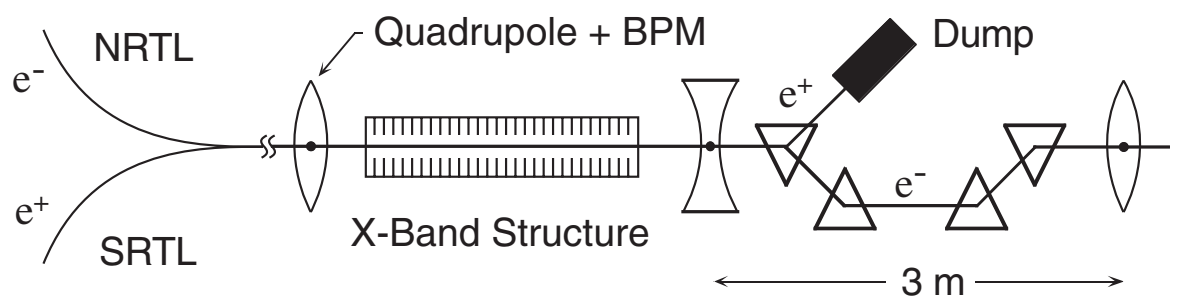

FIG. 10. Schematic of the accelerator structure wakefield measurement facility ASSeT of the SLC. 


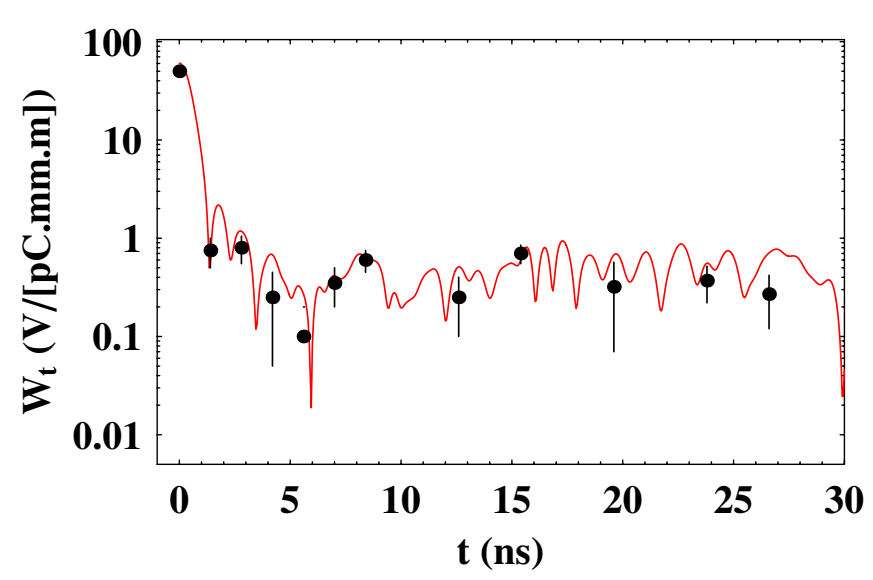

FIG. 11. (Color) Vertical transverse wakefield amplitude in the pair of dipole-mode interleaved accelerator structures. The solid red line is the predicted wakefield and the data points are the experimental measurements.

dence of the wakefield was mapped both locally (over few wavelengths) and globally (over tens of ns in intervals of 1.4 to $8.4 \mathrm{~ns}$ ). The local data were then fit to $15.2 \mathrm{GHz}$ sine waves to determine the wakefield amplitude at the global time separation. These results are plotted in Fig. 11 and compared to predictions (note that the amplitude of the wakefield represents the maximum excursion wakefield that would be witnessed by trailing bunches, so it is the worst-case field). At the level of the measurement errors, there is remarkable agreement, which confirms the precision by which the structures were fabricated and the validity of the wakefield modeling.

The next section discusses how the wakefield affects the low-emittance NLC beams as they traverse the linac. As part of these studies, relatively large random dipole-mode frequency errors are introduced to ascertain whether the cell fabrication tolerances can be relaxed to make them less expensive.

\section{BEAM DYNAMICS}

The progress of the beam down more than five thousand of these accelerating structures will determine whether or not the damping of the wakefield is sufficient to prevent BBU and significant emittance dilution [16,17]. Here we simulate the progress of the beam down a complete linear collider using the code LIAR [18]. We incorporate random frequency errors into a set of 50 accelerating structures and randomly distribute them along the entire linac. In all cases the beam is injected into the linac with an offset of approximately one $\sigma_{y}$, with an energy of $5 \mathrm{GeV}$ and the progress of the beam is monitored as it traverses the entire linac. The final emittance dilution, together with the rms of the sum wakefield, is illustrated in Fig. 12 for small changes in the bunch spacing. The particular simulation illustrated includes a cell-to-cell frequency error with an rms value of $20 \mathrm{MHz}$. We chose this rather large frequency

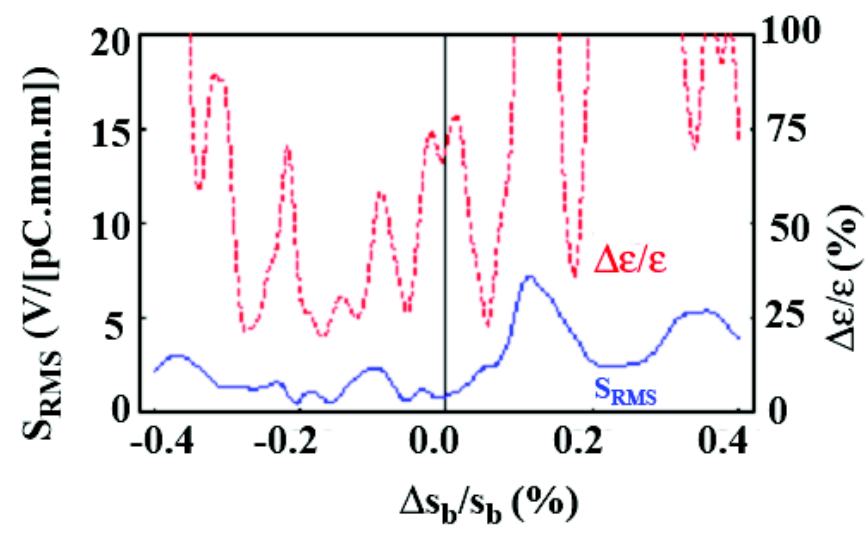

FIG. 12. (Color) Emittance dilution (illustrated by the red dashed curve) versus the percentage change in the bunch spacing. Also shown is the corresponding rms of the sum wakefield (by the solid blue curve).

error in order to gain an understanding of the impact of relaxed manufacturing tolerances on the design of the linac. At the nominal bunch spacing of $1.4 \mathrm{~ns}$, the emittance dilution is approximately $70 \%$. This is reduced by a factor of 2 or more if the bunch spacing is changed by a small fractional amount. For example, if the bunch spacing is increased by $\sim 0.05 \%$, the emittance dilution is reduced to $\sim 25 \%$ and if the bunch spacing is reduced by $0.055 \%$ the emittance dilution is reduced to $28 \%$. These small changes in the bunch spacing correspond to a shift in all the dipole frequencies of the accelerator structure. Thus, the emittance dilution for a $\pm 0.05 \%$ change in the bunch spacing can be achieved by shifting the dipole-mode frequencies by $\sim 7.6 \mathrm{MHz}$. In practice, this is readily achieved by incorporating slight changes in the geometrical parameters of the iris. A further means to minimize the emittance dilution is to introduce emittance bumps into the linac [19]. Nonetheless, the present nonoptimized twofold interleaved set of structures gives a final emittance (with suitable shifting of the mode frequencies) of $\sim 25 \%$ which is close to the NLC emittance budgeted value of $10 \%$ for long-range wakes in the main $X$-band linacs.

\section{CONCLUSIONS}

The experimentally determined values of the wakefield in a pair of $X$-band structures have been shown to be well predicted by the circuit model and spectral function technique. For a linear collider, we would make small changes in the geometry of the accelerating structure in order to fully optimize the spectral function distribution. Furthermore, we also have a carefully optimized design, suitable for linear collider applications that incorporates fourfold interleaving of the dipole frequencies of a group of four structures. This fourfold interleaved design gives rise to no more than $2 \%$ dilution in the final emittance at the end of the $X$-band linacs. Armed with the results of this experimental confirmation of the prediction of wakefield 
for twofold interleaved structures allows us to have confidence in the behavior of fourfold interleaved structures. Furthermore, considerably relaxed tolerances on the randomness of the dipole frequencies have been shown to have minimal negative impact on the emittance dilution of the beam at the exit of the linac. This will allow relaxed tolerances in the cell parameters and will result in considerable savings in the fabrication of several thousand of these structures. The general method of wakefield damping described is applicable to the CLIC scheme, and indeed to any high current low-emittance accelerator.

\section{ACKNOWLEDGMENTS}

The overall program of $X$-band accelerators for linear collider has benefited from seamless collaborations with the following laboratories: SLAC, KEK, Fermi, and LBNL. In particular, we would like to single out the important contributions made by several individuals: N. Baboi, K. Bane, G. Bowden, D. L. Burke, J. Cornuelle, S. Doebert, V. Dolgashev, Z. Farkas, J. Frisch, E. Garwin, S. Harvey, H. Hoag, K. Jobe, R. Kirby, N. Kroll, F. Le Pimpec, G. A. Loew, J. Lewandowski, R. J. Loewen, D. McCormick, R.H. Miller, C. Nantista, J. Nelson, C. K. Ng, R. Palmer, E. Paterson, C. Pearson, N. Phinny, T. Raubenheimer, M. Ross, R. D. Ruth, T. Smith, S. Tantawi, K. Thompson, P. B. Wilson, SLAC; H. Baba, Y. Funahashi, N. Higashi, Y. Higashi, T. Higo, N. Hitomi, H. Kawamata, N. Kudo, T. Kume, H. Matsumoto, Y. Morozumi, J. S. Oh, T. Shintake, K. Takata, T. Takatomi, N. Toge, K. Ueno, Y. Watanabe, KEK; T. Arkan, C. Boffo, H. Carter, D. Finley, I. Gonin, T. Khabiboulline, S. Mishra, G. Romanov, N. Solyak, FNAL; J. Klingmann, K. van Bibber, $L L N L$. Furthermore, we are pleased to acknowledge the instrumental modifications made to the computational code LIAR, by Dr. Peter Tenenbaum. Without his aid, the simulations discussed in the penultimate section would not have been possible. We received assistance in data taking from Dr. Mauro Pivi and Dr. Stefan Doebert.
This work is supported by the U.S. Department of Energy under Contract No. DE-AC02-76SF00515.

[1] International Linear Collider Technical Review Committee (ILC-TRC) Second Report, 2003, SLAC-R606.

[2] International Linear Collider Technical Review Final Report, 2004 (http://www.linearcollider.org).

[3] The TESLA Technical Design Report, edited by R. Brinkman et al., 2001, DESY Report 2001-33.

[4] R. R. Larsen and G. A. Loew, SLAC-TN-62-57, 1962.

[5] C. Adolphsen, presented at the 2005 Particle Accelerator Conference (PAC 05), Knoxville, Tennessee, 2005.

[6] K. L. F. Bane, P. B. Wilson, and T. Weiland, AIP Conf. Proc. 127, 875 (1985).

[7] K. L. F. Bane, SLAC-PUB-9663, 2003.

[8] X. Zhan, Ph.D. thesis, Stanford University, 1997.

[9] H. Braun et al., Updated CLIC parameters 2005, CLIC note 627, 2006.

[10] J.-Y. Raguin, D. Schulte, I. Syratchev, I. Wilson, and W. Wuensch, Proceedings of LINAC2002, Gyeongju, Korea, 2002.

[11] The MAFIA Collaboration, MAFIA-L, CST, GmbH, Darmstadt, 1994.

[12] Z. Li et al., presented at the 9th European Particle Accelerator Conference (EPAC04), Lucerne, Switzerland, 2004.

[13] K. L. F. Bane and R. L. Gluckstern, Part. Accel. 42, 123 (1993).

[14] P. B. Wilson, SLAC-PUB-4547; SLAC-AP-66; SLAC-AP066, 1989.

[15] C. Adolphsen et al., Phys. Rev. Lett. 74, 2475 (1995).

[16] K. L.F. Bane, C. Adolphsen, K. Kubo, and K. A. Thompson, The 4th European Particle Accelerator Conference (EPAC94), London, England, 1994.

[17] K. Thompson and R.D. Ruth, Phys. Rev. D 41, 964 (1990).

[18] R. Assman et al., SLAC-PUB AP-103, 1997.

[19] P. Tenenbaum, LCC-Note-0138, 2004. 